\title{
Acute lung injury: how the lung inflammatory response works
}

\author{
P.A. Ward
}

The purpose of this article is to provide an overview of the lung inflammatory response in rodents. Topics to be discussed will be initiation of the inflammatory response [1], inflammatory products that are responsible for tissue damage, and the intrinsic pathways that regulate (depress) the inflammatory response leading to its containment [2].

\section{Acute inflammatory response}

Acute lung injury in rodents (mice, rats) can be induced by intrapulmonary deposition of immunoglobulin (Ig)G immune complexes, which trigger an intense inflammatory response characterised by the influx of polymorphonuclear neutrophils (PMN), interstitial and alveolar oedema, and intra-alveolar haemorrhage [3]. This model of acute inflammation has been extensively evaluated in order to understand how the acute inflammatory response is triggered. This inflammatory response is dependent on generation of the powerful complement activation product C5a. Generation of C5a in lung can be accomplished by activation of any one of the three pathways of complement activation (classical, alternative and lectin pathways). An altogether different pathway for $\mathrm{C} 5 \mathrm{a}$ generation in lung involves cleavage of $\mathrm{C} 5$, which is produced by lung cells, by a serine protease released from activated alveolar macrophages. C5a, together with $\mathrm{IgG}$ immune complexes, causes activation of lung macrophages, with resultant production of cytokines (e.g. interleukin (IL)-1 and -6 , tumour necrosis factor (TNF)- $\alpha$ ) and chemokines (CXC chemokines such as macrophage inflammatory protein (MIP)-2 and KC and CC chemokines such as MIP- $1 \alpha$ and MIP-1 $\beta$ ). These products participate in several important functions. IL-1 and TNF- $\alpha$ cause upregulation of adhesion molecules on the pulmonary vascular endothelium (E-selectin, intercellular adhesion molecule (ICAM)-1) as well as on the alveolar epithelium (ICAM-1). Vascular adhesion molecules enhance adhesiveness of PMN and facilitate their subsequent transmigration into the interstitial and distal airways of lung. Alveolar epithelial ICAM-1 enhances adhesiveness of alveolar macrophages to the epithelial cells, resulting in intensified production of cytokines and chemokines. Production of IL-6 during this inflammatory process causes upregulation of $\mathrm{C} 5 \mathrm{aR}$ on vascular endothelial cells, and alveolar epithelial cells. This facilitates interaction with $\mathrm{C} 5 \mathrm{a}$, which enhances cell function and production of proinflammatory mediators. Production of CXC chemokines within the lung results in chemoattraction of PMN into the lung interstitial and alveolar compartments. The CC chemokines function as autocrine factors that positively enhance

Correspondence: P.A. Ward, Dept of Pathology, University of Michigan Medical School, M5240 Medical Science I Bldg, 1301 Catherine Rd, Ann Arbor, MI 48109-0602, USA. Fax: 17347634782. E-mail: pward@umich.edu macrophage function, resulting in enhanced generation of CXC chemokines.

Products of the acute inflammatory response in lung causing tissue injury are released from recruited PMN and activated lung macrophages [4]. These include toxin oxygen $\left(\mathrm{O}_{2}\right.$, hydrogen peroxide, etc. $)$ and nitrogen (nitrosyl, peroxynitrite, etc.) products, together with proteases released from these phagocytic cells. Under certain conditions C5a can interact with cells containing upregulated $\mathrm{C} 5 \mathrm{aR}$, with resulting caspase activation and apoptosis. This appears to be the case especially with lymphoid cells.

The acute inflammatory reaction in lung depends on nuclear transcription factor (NF)- $\kappa \mathrm{B}$ activation, which results in activation of proinflammatory genes, with release of the cytokines and chemokines that are described above. As the inflammatory reaction progresses, regulatory (inhibitory) gene products also appear, especially IL-10 and IL-13. These ILs are powerful anti-inflammatory mediators that suppress NF- $\kappa \mathrm{B}$ activation. This is due to the fact that they prevent hydrolysis of $\mathrm{I} \kappa \mathrm{B}-\alpha$, which must be broken down if $\mathrm{NF}-\kappa \mathrm{B}$ activation is to occur. Under such conditions, the NF$\kappa \mathrm{B}$ complex (p50, p65), which is interlocked with $\mathrm{I} \kappa \mathrm{B}-\alpha$ protein, cannot be translocated to the nucleus, preventing gene activation. During the acute inflammatory response in lung, both IL-10 and IL-13 proteins appear. If either is blocked with relevant antibody, the inflammatory response is intensified. Another regulatory protein that is generated during the acute inflammatory response is the secreted leukocyte protease inhibitor (SLPI). This protein also inhibits $\mathrm{NF}-\kappa \mathrm{B}$ activation by causing high cytoplasmic levels of I $\kappa \mathrm{B}-\beta$, the presence of which also prevents translocation of $\mathrm{NF}-\kappa \mathrm{B}$ to the nucleus. If antibody to SLPI is instilled into the lung, the inflammatory response is intensified, providing evidence that SLPI functions as a natural regulator of the lung inflammatory response. Thus, at least three naturally produced factors (IL-10, IL-13, SLPI) during the acute inflammatory response function to contain the inflammatory response.

These data define the complex pathways that lead to acute lung inflammatory injury. The mediators responsible for triggering the influx of PMN are numerous. The functional effects of these mediators can be classified as initiators and propagators of the inflammatory response, as described above. At the same time that proinflammatory mediators are being generated, anti-inflammatory mediators are also being produced. The balance between these two classes of mediator determines whether the inflammatory response persists and intensifies, or whether it is contained.

\section{Conclusion}

Initiation of acute inflammatory injury in rodent lungs is initiated by a complex series of events, with production of proinflammatory mediators that activate adhesion 
molecules (for leukocytes) on the vascular endothelium and on the distal airway epithelium, resulting in recruitment of polymorphonuclear neutrophils into the alveolar compartment and activation of lung macrophages. Several natural inhibitors are also produced during these inflammatory reactions. These inhibitors work chiefly by preventing nuclear factor- $\kappa \mathrm{B}$ activation, which suppresses production of proinflammatory mediators. The outcome of the inflammatory response (progression or containment) depends upon the balance between proinflammatory and anti-inflammatory mediators. The extent to which similar inflammatory mechanisms operate in the context of allergic responses and in chronic obstructive pulmonary disease conditions is not clear.

\section{References}

1. Collins T. Acute and chronic inflammation. In: Cotran RS, Kumar V, Collins T, eds. Robbins: Pathologic Basis of Disease. 6th Edn. Philadelphia, WB Saunders Company, 1999; pp. 50-88.

2. Lentsch AB, Ward PA. Regulation of experimental lung inflammation. Respir Physiol 2001; 128: 17-22.

3. Guo RG, Ward PA. Mediators and regulation of neutrophil accumulation in inflammatory responses in lung: insights from the IgG immune complex model. Free Rad Biol Med 2002; 33: 303-310.

4. Sacca R, Cuff CA, Ruddle NH. Mediators of inflammation Curr Opin Immunol 1997; 9: 851-857. 